\title{
Uso de tecnologias de información y comunicación en el screening de la depresión asociada al consumo de sustancias psicoactivas
}

\author{
Use of information and communication technologies in screening of \\ depression associated with the use of psychoactive substances
}

Edith Villalobos Bolivar¹, Luis Gilberto Agreda López², Ángel Alberto Varón Quimbayo³

\section{Resumen}

Introducción. El consumo de sustancias psicoactivas (SPA) se consideran como una enfermedad crónica debido a que el inicio, curso, dirección, y resultado es impredecible en algunos casos y adicionalmente son trastornos multicausales como es el caso de la depresión; según datos reportados por el Centro de Adicciones de Barcelona "la incidencia de depresión en pacientes con abuso de sustancias oscila entre el 6\% y 70\% en los consumidores de SPA". Con este panorama se debe comprender que la intervención debe desarrollar la promoción de la salud y la prevención de la enfermedad, con la aparición de la biotecnología con el uso y aplicación de las tecnologías de la información y las comunicaciones (TIC) en las ciencias de salud y la vida para el diagnóstico, prevención, supervisión, tratamiento. Objetivo. Desarrollar un software que permita generar una alerta para la identificación temprana de la depresión asociada al consumo de sustancias psicoactivas en adultos, mediante el uso de una TICs previamente validada, durante el año 2019. Metodología. Investigación Tecnológica, utilizando la metodología "UWE" con enfoque al desarrollo de software para el dominio web. Resultados. En esta propuesta de investigación se avanzará en el desarrollo del prototipo del producto para la identificación temprana de la depresión con el fin de mejorar el proceso de toma de decisiones clínicas, en el momento mismo de la atención,

\footnotetext{
1. MsC Salud Pública y Desarrollo Social. Docente Fundación Universitaria del Área Andina. Programa Enfermería. ORCID: https://orcid.org/0000-0002-5993-5404

CvLac: https://scienti.colciencias.gov.co/cvlac/visualizador/generarCurriculoCv.do?cod rh $=0001599196$

Google Scholar: https://scholar.google.es/citations?hl=es\&pli=1\&user=mIHdHAcAAAAJ

2. MsC Salud Pública y Desarrollo Social. Docente Fundación Universitaria del Área Andina. Programa Enfermería. Coordinador Semillero Conexión Salud - Grupo de Investigación Guanaco. ORCID: https://orcid.org/0000-0002-8648-8267

CvLac: https://scienti.minciencias.gov.co/cvlac/visualizador/generarCurriculoCv.do?cod_rh $=0001466332$

Google Scholar: https://scholar.google.es/citations?pli=1\&user=0PToMXgAAAJ

3. Mag Gestión Diseño y Dirección de Proyectos. Docente Fundación Universitaria del Área Andina. Programa Ing de Sistemas ORCID: https://orcid.org/0000-0003-0643-358X

CvLac: https://scienti.minciencias.gov.co/cvlac/visualizador/generarCurriculoCv.do?cod_rh=0000012309

Google Scholar: https://scholar.google.es/citations?user=52pp2VQAAAJ\&hl=es
}

Correo electrónico de correspondencia: glvillalobos@areandina.edu.co - lagreda@areandina.edu.co - avaron2@areandina.edu.co 
con base en la información registrada en el software llamado MEASDEP (Medición de la depresión en consumidores de SPA).

Palabras claves: sustancias psicoactivas (SPA), depresión, consumo, biotecnología, tecnología de la información y la comunicación (TIC).

\section{Abstract}

Introduction. The consumption of psychoactive substances (SPA) is considered a chronic disease because the onset, course, direction, and result is unpredictable in some cases and additionally they are multi-causal disorders such as depression; according to data reported by the Barcelona Addiction Center "the incidence of depression in patients with substance abuse ranges between $6 \%$ and $70 \%$ in SPA users". With this outlook, it should be understood that the intervention should develop the promotion of health and the prevention of disease, with the appearance of biotechnology with the use and application of information and communication technologies (ICT) in science, health and life for diagnosis, prevention, supervision, treatment. Objective. To develop a software that allows generating an alert for the early identification of depression associated with the use of psychoactive substances in adults, through the use of a previously validated ICT, during 2019. Methodology. Technological Research, using the "UWE "With a focus on software development for the web domain. Results. In this research proposal, progress will be made in the development of the product prototype for the early identification of depression in order to improve the clinical decision-making process, at the moment of care, based on the registered information in the software called MEASDEP (Measurement of depression in SPA consumers)

Keywords: psychoactive substances (SPA), depression, consumption, information and communication technology (ICT), medical informatics.

\section{Introducción}

El consumo de sustancias psicoactivas en adultos jóvenes es un problema de salud pública que ha tenido un crecimiento marcado en los últimos años. Según el Informe Mundial de Drogas 2018 de UNODC, un cuarto de billón de personas o cerca del 5\% de la población adulta, entre los 15 y los
64 años, consumió drogas por lo menos en una ocasión; es de aclarar que a partir del 2015 (1) se considera droga toda sustancia química que entre en contacto con los diferentes sistemas del cuerpo humano produciendo efectos a corto y largo plazo que se traducen clínicamente en la presencia de un síndrome de abstinencia si no se utiliza la droga y de tolerancia progresiva a su uso 
generando un impacto negativo en el desarrollo humano, la salud y la calidad de vida de los consumidores.

En cuanto a los efectos negativos, que en su mayoría se asocian al post consumo, el consumidor puede experimentar diversos efectos adversos acorde como la sustancia interaccione con el cuerpo humano y sus diferentes sistemas provocando alteraciones que pueden ser fatales. La depresión asociada al consumo de sustancias psicoactivas es uno de los efectos más comunes en la población consumidora de Sustancias Psicoactivas (SPA) (2), la morbilidad de los trastornos psiquiátricos en general y de los trastornos afectivos o depresivos asociados al consumo de drogas y alcohol se ha multiplicado en los últimos 10 años (3). Uno de los trastornos más comunes que afectan la salud mental es la ansiedad y los trastornos de ansiedad tienen relación directa con los problemas de violencia, la pobreza y el consumo de sustancias psicoactivas como estresantes de la sociedad en que vivimos (4). Los síntomas psiquiátricos negativos se conciben como una dimensión característica de diferentes trastornos e incluyen la afectividad negativa y el déficit cognitivo.

En la actualidad los trastornos por consumo de SPA se consideran como una enfermedad crónica debido a que el inicio, curso, dirección, y resultado son impredecibles en algunos casos. Según datos reportados por el Ministerio de Salud, uno de los factores causales del riesgo de depresión en pacientes, está asociado al abuso y la dependencia de las sustancias psicoactivas incluidos los consumidores de alcohol y cigarrillo(5); con este panorama se debe comprender que la intervención debe ser integral e integra donde la aparición de las tecnologías de la información y las comunicaciones (TIC) en salud son vitales para el diagnóstico, prevención, supervisión, tratamiento o alivio de una enfermedad; como lo menciona el Decreto 4725 de 2005.

En este sentido las Tecnologías de la Información y la Comunicación, también conocidas como (TIC), son el conjunto de tecnologías desarrolladas para gestionar información y enviarla de un lugar a otro y abarcan un abanico de soluciones amplio, las cuales han evolucionado para acceder y compartir información en la Web como también han permitido el desarrollo de sistemas adecuados para ubicarlos en línea y fuera de línea para la investigación colaborativa, asincrónica y de discusión para aumentar la generación y trasferencia de conocimientos(6). Las TIC incluyen las tecnologías para almacenar información y recuperarla después, enviar y recibir información de un sitio a otro, o procesar información para poder calcular resultados y elaborar informes (7).

Por tanto, esta investigación se enfoca en identificar las herramientas, las técnicas, la metodología y los IDE (entornos de desarrollo integrados), adecuados para desarrollar un producto de software orientado a la web para la medición de la depresión y que genere alertas tempranas en la población de adultos consumidores de SPA inicialmente en la ciudad de Bogotá, 


\section{Materiales y metodos}

Metodología: Investigación Tecnológica. UWE (UML-BASED WEB ENGINEERING)

"UWE" es una metodología apoyada en el desarrollo de software con enfoque de la ingeniería del software para el dominio web, la cual utiliza un lenguaje de modelado específico del dominio basado en UML Lenguaje unificado para modelamiento de sistemas de información (software), los Modelos de UWE son una extensión de UML que presenta los siguientes modelos:

- Modelo de requerimientos donde se hace la especificación de requerimientos del software y se generan los modelos de casos de uso donde se ilustra la funcionalidad del producto,

- El Modelo de contenido es un modelo normal donde se grafican las clases don- de expresa el dominio o la arquitectura de cada atributo en su respectiva clase

- El modelo de navegación o navegabilidad se realiza para representar en modo grafico cada uno de los nodos con su respectivo link para facilitar el entendimiento de la forma en que se podrá navegar por el software, para hacerlo más amigable y con esto apuntarle al criterio de usabilidad.

- El Modelo de presentación se realiza con el objetivo de representar los elementos que serán utilizados en cada uno de los índex del software (botones, textos, formularios, imágenes, entradas de texto entre otras).

- El Modelo de procesos representa de forma gráfica las relaciones existentes entre las diferentes clases, para ilustrar la funcionalidad dentro del producto (8).

Figura 1. Fases del proceso investigativo.

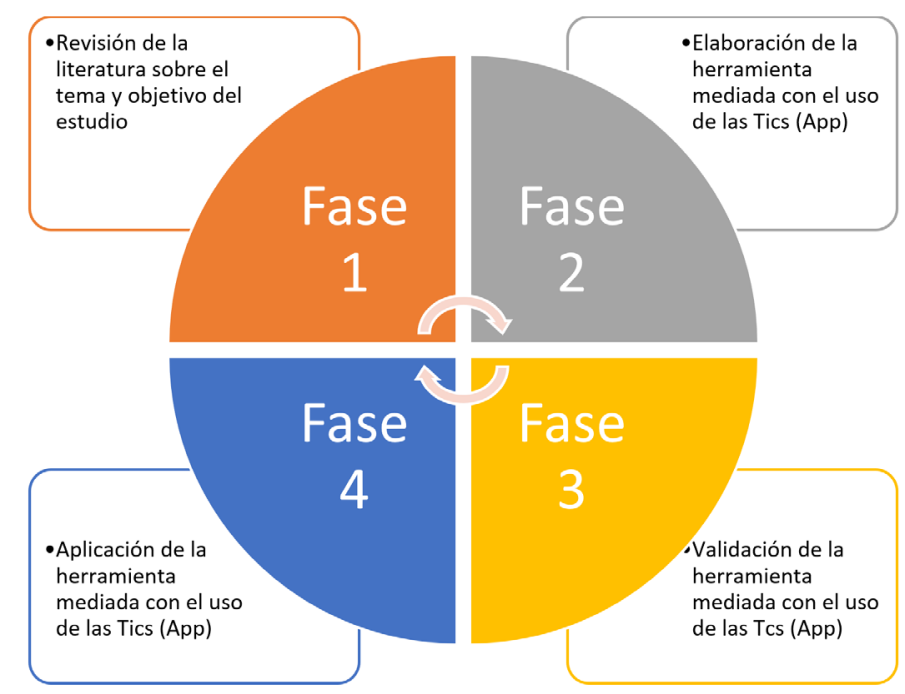

Fuente: Elaboración propia. 
FASE 1: Revisión de la literatura para implementar la metodología UWE para el desarrollo del software Measdep para la identificación temprana de la depresión en adultos consumidores de SPA.

FASE 2: Diseño y desarrollo del software Measdep para la identificación de la depresión en adultos consumidores de SPA.

FASE 3: Testing pruebas del software Measdep para validar la funcionalidad del software según los requerimientos establecidos

FASE 4: Implementación del software Measdep para la identificación de la depresión en adultos consumidores de SPA

La metodología UWE es una extensión del lenguaje unificado para modelo de sistemas de información UML y se enfoca única y exclusivamente a desarrollos orientados a la web, para realizar unos muy buenos diseños se recomienda utilizar la herramienta CASE (Diseño asistido por computadora) Magic UWE que fue desarrollada para facilitar el trabajo al profesional de diseño (9).

Para el desarrollo de software seleccionamos el entorno de desarrollo XAMP que es una plataforma gratuita distribuida por apache que es un servidor local y que dentro de sus herramientas cuenta con el lenguaje PHP y un sistema gestor de bases de datos Mysql para el desarrollo de la base de datos, también se utiliza CSS la hoja de estilo en cascada para mejorar la presentación del producto como tal, para la visualización en los diferentes tipos de dispositivo móvil se utiliza una técnica Responsive que consiste en la adaptación de contenidos web a dispositivos móviles para facilitar su usabilidad.

\section{Resultados}

En búsqueda de automatizar procesos de tamizaje para la depresión en consumidores de sustancias psicoactivas, se desarrolla un software para la medición y la evaluación de los consumidores de spa. Para el desarrollo de dicho instrumento se trabaja de manera conjunta el área del programa de enfermería y del programa de ingeniería de sistemas de la Fundación Universitaria del Área Andina apoyándose en la implementación de la Ingeniería web, adaptando la metodología UWE; durante el proceso investigativo se plasman las bases y referentes teóricos para definir las características del diseño de la aplicación el cual será utilizado por el área de ingeniería de sistemas la Fundación Universitaria del Área Andina que elabora la especificación de requisitos de software.

MEASDEP es el nombre que recibe el software donde se reconocerá y creará usuarios para la captación de información personal con el objetivo de brindar apoyo que permita la identificación temprana de la depresión asociada al consumo 
de SPA; adicionalmente permite gestionar el instrumento de tamizaje seleccionado, el cual cuenta con un cuestionario de 10 preguntas donde cada una de ellas se identifica con un número original y tiene su respectivo enunciado los cuales una vez diligenciadas permite generar resultados y puntuaciones para posteriores recomendaciones pertinentes referenciadas por la Guía de Práctica Clínica
El software MEASDEP como instrumento, brindara soporte a enfermeros profesionales de la Fundación universitaria del Área Andina para detectar la depresión temprana en adultos y adolescentes, consumidores de sustancias psicoactivas SPA.

Figura 2. Pantalla de inicio de la aplicación.
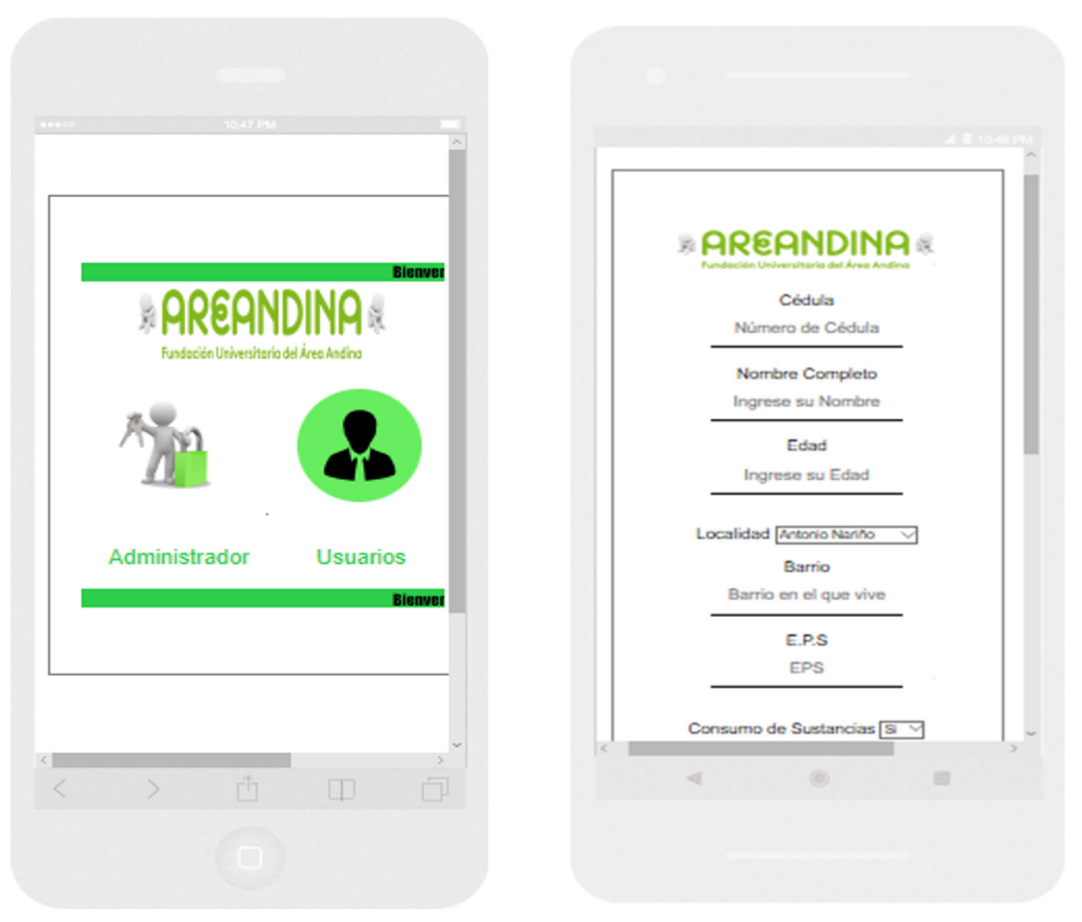

Fuente: Elaboración propia.

\section{Discusión}

Los avances tecnológicos fácilmente pueden ser vistos como una revolución que representa la creación de oportunidades y desafíos sin precedentes en el área de las ciencias de la salud; de igual forma el uso de las TIC es clave para agilizar procesos y procedimientos, también; elimina barreras y permite reducir las desigualdades y superar la pobreza, asegurando un cuidado integral a una población.

Según la Organización Mundial de la Salud (OMS), la cibersalud (conocida tam- 
bién como eSalud o e-health) consiste "en el apoyo que la utilización de las tecnologías de la información y las comunicaciones TIC, brinda a la salud y a los ámbitos relacionados con ella, con inclusión de los servicios de atención de salud, la vigilancia y la documentación sanitarias, así como la educación, los conocimientos y las investigaciones en materia de salud".

Mediante el uso de estas tecnologías se busca abordar la prevención, el diagnóstico, el tratamiento y el monitoreo o incluso la administración de los sistemas sanitarios. En la actualidad contamos con diversas aplicaciones como: - "Social Diabetes" lo cual es una herramienta de ayuda al control de la diabetes tipo 1 y 2 , permite a los pacientes calcular las dosis de hidratos y la administración de insulina; - "Universal Doctor" la cual es conocida en algunos países como Universal Doctor Speaker tratándose de una aplicación que busca servir como traductor médico que facilita las consultas tanto a profesionales de la salud como a pacientes; -"Foto Skin" cuya aplicación es el resultado de investigación desarrollada por la Universidad de Alcalá de Henares, en Madrid, España, la cual sirve de guía para pacientes y médicos para el diagnóstico del cáncer de piel y consiste en un portal web, con un tutorial, donde se aprende a tomar las imágenes para formar un registro e indicar el fototipo de piel del usuario lo cual ya puede ayudar en cuanto a la prevención de la enfermedad". Todas estas aplicaciones permiten al personal de salud y a los pacientes un acceso a la salud solo con el uso de su dispositivo móvil.
Si bien existe variedad de aplicaciones, aún no se cuenta con un software adaptable a dispositivos móviles que permita la detección de la depresión asociada al consumo de SPA, por lo tanto, se debe reconocer la innovación que esta investigación ofrece. MEASDEP como software permitirá al usuario el registro voluntario para captar información personal con el objetivo de generar una alerta temprana de los estados depresivos asociados al consumo de SPA. La herramienta cuenta con un cuestionario de 10 preguntas que permitirán identificar usuarios con depresión acorde a resultados y puntuaciones, brindando soporte para posteriores tomas de decisiones referenciadas por la Guía de Práctica Clínica (5).

\section{Conclusiones}

La revisión bibliográfica dentro del contexto de instrumentos utilizados para realizar el tamizaje para detectar depresión determino que dentro de los instrumentos que se utilizan en el marco comunitario la mejor opción es el CES-D debido a que es auto administrado y permite medir la depresión en 4 ocasiones y así poder determinar un riesgo de depresión. Se ha diseñado y desarrollado el software con orientación a la web MEASDEP, y mediada por el uso de las Tics usando la escala CES-D, la cual permitirá identificar a la población con riesgo a la depresión o con depresión asociada al consumo de sustancias psicoactivas siendo esta capaz de recolectar información e interactuar con el usuario consumidor de spa. 
Se espera que en el segundo semestre de 2019 se lleve a cabo la implementación del software Measdep, herramienta mediada por el uso de las Tics la cual cuenta con la documentación especifica de la ingeniería del software Web, según la metodología UWE: (Documento de E.R.S, los diagramas casos de uso, el modelo de contenido, el modelo de navegabilidad, el modelo de presentación y el modelo de Procesos), más el software desarrollado, e implementado con el objeto de iniciar el tamizaje en la población de localidad de Chapinero en adultos jóvenes consumidores de sustancias psicoactivas

\section{Referencias}

1. Oficina de las Naciones Unidas contra la Droga y el Delito (UNODC). Informe Mundial sobre las Drogas. [Internet].; 2018. Disponible en: https:// www.unodc.org/wdr2018/prelaunch/WDR18_ExSum_Spanish.pdf

2. Gainza I, Nogué S, Martínez Velasco C, Hoffman RS, Burillo-Putze G, Dueńas A, et al. Intoxicacion por drogas. Anales Sis San Navarra vol.26. [Internet]. 2003. Disponible en: https://recyt.fecyt.es/ index.php/ASSN/article/view/5014/4252

3. Bohnert S, Miech A. Changes in the Association of Drug Use with Depressive Disorders in Recent Decades: The Case of Cocaine. Substance Use \& Misuse. 2010;: p. Vol. 45 Issue 10, p1452-1462. . [Internet]. 2010. Disponible en: https://www.ncbi. nlm.nih.gov/pmc/articles/PMC4109050/

4. Posada-Villa J, Rodríguez M, Duque P, Garzón A. Prevalencia y factores de riesgo de comportamientos relacionados con suicidio en el Estudio Nacional de Salud Mental, Colombia 2003 (ENSM). NOVA [Internet]. 30jun.2007 [citado 28sep.2020];5(7). Available from: https://revistas. unicolmayor.edu.co/index.php/nova/article/view/ 87Bohnert S, Miech A. Changes in the Association of Drug Use with Depressive Disorders in Recent Decades: The Case of Cocaine. Substance Use \& Misuse. 2010;: p. Vol. 45 Issue 10, p1452-1462. . [Internet]. 2010. Disponible en: https://www.ncbi. nlm.nih.gov/pmc/articles/PMC4109050/

5. Posada-Villa J, Buitrago-Bonilla J, Medina-Barreto Y, Rodríguez-Ospina M. Trastornos de ansiedad según distribución por edad, género, variaciones por regiones, edad de aparición, uso de servicios, estado civil y funcionamiento/discapacidad según el Estudio Nacional de Salud Mental-Colombia. NOVA [Internet]. 31dic.2006 [citado 5oct.2020];4(6). Available from: https://revistas.unicolmayor.edu. co/index.php/nova/article/view/67

6. Ministerio de Salud y Protección Social y Colciencias (2013) Guía de Práctica Clínica Depresión, volumen 22. [Consultado el 23 de enero de 2018]. Disponible en: http://gpc.minsalud.gov.co/ gpc_sites/Repositorio/Conv_500/GPC_td/GPC_ td_completa.aspx

7. Millan J, Yunda L. An Open-Access Web-based Medical Image Atlas for Collaborative Medical Image Sharing, Processing, Web Semantic Searching and Analysis with Uses in Medical Training, Research and Second Opinion of Cases. NOVA [Internet]. 31 dic.2014 [citado 28sep.2020];12(22). Available from: https://revistas.unicolmayor.edu.co/index. $\mathrm{php} /$ nova/article/view/273

8. Universidad de Antioquia. Aprende en Línea Plataforma Academica para Investigación. [Internet].; 2015. Disponible en: http://aprendeenlinea. udea.edu.co/lms/investigacion/mod/page/view. php?id $=3118$

9. Institute for Informatics. Universitat München UWE - UML-based Web Engineering (18 de 06 de 2016). [Internet]. Disponible en: http://uwe.pst. ifi.lmu.de/toolUWE4JSF.html 